\title{
HIV/AIDS and psychiatry: Towards the establishment of a pilot programme for detection and treatment of common mental disorders in people living with HIV/AIDS in Cape Town
}

As the roll-out of antiretrovirals (ARVs) to people living with HIV/AIDS (PLWHA) continues to increase in South Africa, so too does the need to integrate mental health services into HIV care. In this editorial, we argue that the role of mental health in ARV programmes is central. The prevalence of mental disorders in PLWHA is higher than in the general population, and the impact of these conditions is substantial. Screening tools for mental disorders are both available and feasible. These should be incorporated into routine ARV care, with support from dedicated HIV mental health services.

In PLWHA there is growing evidence of an increased prevalence of mental disorders compared with the general population. For example, in a meta-analysis of 10 studies, major depression was nearly twice as common in PLWHA. 'Similarly, in the HIV Cost and Services Utilization Study (HCSUS) conducted in the $U_{S A}{ }^{2}$ the prevalence of depressive, anxiety and substance use disorders in PLWHA was at least twice that reported in the NCS-R. In the HCSUS, the prevalence of alcohol and substance abuse combined was reported as $50.1 \%$, while neurocognitive disorders affect as many as 30\% of PLWHA, particularly in late stages. ${ }^{3}$ Mental disorders found to be highly prevalent in a South African ARV clinic include major depression (34.9\%), posttraumatic stress disorder (14.8\%) and alcohol abuse (10.1\%). ${ }^{4}$

In addition to these mental disorders, neurocognitive disorders (NCDs) are emerging as a new challenge in the era of ARVs. They often persist despite the use of $A R V_{s}$, with consequent neurodegeneration. ${ }^{5} \mathrm{NCDs}$, with the typical sub-cortical pattern, are being encountered increasingly in ARV clinic populations. Frank HIV dementia (HIV-D) may be occurring less frequently than before, but as the duration of illness increases with ARVs, it is now recognised that prevalences may actually be increasing. ${ }^{6}$ Minor forms of NCD also occur, and with greater frequency than HIV-D. While these forms of neurological disease are less disabling, they nonetheless also result in a significant burden of disease. ${ }^{5}$

While it is known that NCDs are highly prevalent, and that minor forms are often not detected, a unique opportunity exists to address some of these questions. While all patients entering ARV care should be screened for NCD, the issue is whether or not individuals with CD4 counts greater than 200 cells $/ \mu$ and demonstrable neurocognitive impairment should be commenced on $A R V$ s for their relative neuroprotective effects. How busy primary care clinics integrate this screening into routine care should be discussed and researched.

The impact of mental disorders on outcomes in PLWHA is now undisputed. They affect adherence, mortality and quality of life. ${ }^{7}$ In addition, many mental disorders increase the probability of engaging in risk behaviours, ${ }^{8}$ and are aggravated by or caused by ARV treatment. ${ }^{9}$ In the context of the large numbers of PLWHA in South Africa who are infected and who access ARV care, this burden is substantial. South Africa has the largest number of people infected with HIV worldwide. ${ }^{10}$ With South Africa deploying one of the more successful ARV roll-outs in the developing world, more than half of PLWHA in the Western Cape will receive ARVs. ${ }^{11}$ These growing numbers of patients accessing HIV care have seen the rates of retention in care falling. Of the cohort commencing ARVs in 2005, 13.8\% were no longer in care at 2 years. ${ }^{11}$ Of this number, approximately $40 \%$ are people lost to follow-up (LTFU). LTFU is a crude proxy measure for poor adherence. Substance abuse and mental disorders are common reasons for poor adherence. ${ }^{12}$

With all of these conditions affecting large numbers of PLWHA, screening programmes for mental illness are increasingly being regarded as essential to HIV care. In South Africa, it is a requirement that patients enrolled onto $A R V_{s}$ receive some form of screening for both depression and alcohol abuse. It is our impression that this is done with varying accuracy and efficacy. While Patel et al. have argued that the training of primary care physicians leads to transient improvements in detection, screening tools may actually improve outcomes in general primary care clinics. ${ }^{13}$ Brief instruments for these settings do exist (such as the General Health Questionnaire and the Kessler-10 item scale), 14,15 but few are aimed at the specific problems encountered in ARV clinic populations. A MEDLINE search for such a tool yielded only the Substance Abuse and Mental Illness Symptoms Screener (SAMISS), a tool validated in PLWHA in the USA. ${ }^{16}$ It includes screening questions for alchohol and substance abuse, major depression, panic disorder and PTSD, as well as adjustment disorder. These are an important group of disorders commonly encountered in the pre-ARV treatment group. Other disorder- 
specific tools have been validated in South Africa, namely the Alcohol Use Disorders Identification Test (AUDIT), the Centres for Epidemiological Study-Depression Scale (CES-D) and the Harvard Trauma Scale (HTS). ${ }^{17}$ The combination of these tools would however prove too lengthy.

Together with the SAMISS, a screen for neurocognitive disorder should be done on all patients commencing ARVs, those failing first-line treatments, and those who have been on ARVs for more than 2 years. The International HIV Dementia Scale ${ }^{18}$ is a suitable brief screening tool for this setting. Together the SAMISS and IHDS screen takes $10-12$ minutes. The sensitivity and specificity of the SAMISS are $97 \%$ and $44 \%$ respectively, ${ }^{16}$ while those of the IHDS were reported to be $80 \%$ and $55 \%$ respectively. ${ }^{18}$ The high sensitivities would normally result in a high degree of case detection, while the moderate specificities may lead to a number of false-positive cases. These should be reviewed at a second tier of screening by staff with a higher level of skills.

In order to implement these interventions as widely as possible, data on suitability and feasibility are needed. Such a pilot programme is underway in the HIV/neuropsychiatry programme in the Western Cape. We suggest that existing lay counsellors or nurses be trained to administer these tools. A study comparing the results obtained by trained mental health professionals and lay interviewers needs to be done to establish levels of agreement. Following this, additional measures, together with an assessment of true case-ness using a semi-structured diagnostic interview (such as the MINI neuropsychiatric interview), should be done to establish the local diagnostic validity and utility of the SAMISS and IHDS. The impact of these tools on clinic function, acceptability to personnel and ease of use should be measured. Cases detected by lay counsellors or nurses should then be referred to a mental health nurse for more detailed assessment. True cases may be managed on site by community mental health services, or referred to secondary support clinics if needed. Information regarding the reasons for referral out should be captured, so as to plan future services and needs.

Resources for HIV and mental health are scarce. To our knowledge, there are very few dedicated HIV psychiatry services in South Africa. The role of HIV psychiatrists should be to develop and co-ordinate services to PLWHA using existing frameworks. Additional support and development should be added. This may take the form of medical officers to manage referred patients, and lay counsellors and nurses to provide primary care screening and treatment. Once feasibility and effectiveness have been shown, the programme could be expanded to reach all PLWHA. These tasks need to shift to the non-psychiatrist. With suitable training and support, it is possible that mental health services can be integrated into routine ARV care. Lessons from this disease may then be transferred into general primary care settings.

\section{John A Joska \\ Dan J Stein \\ Alan J Flisher \\ Department of Psychiatry and Mental Health University of Cape Town}

1. Ciesla JA, Roberts JE. Meta-analysis of the relationship between HIV infection and risk for depressive disorders. Am J Psychiatry 200 1; 158(5): 725-730

2. Bing EG, Burnam MA, Longshore $D$, et al. Psychiatric disorders and drug use among human immunodeficiency virus-infected adults in the United States. Arch Gen Psychiatry 2001 ; 58(8): $721-728$.

3. Grant I. Neurocognitive disturbances in HIV. International Review of Psychiatry 2008 20(1): 33-47

4. Olley B, Seedata S, Stein D. Persistence of psychiatric disorders in a cohort of HIV/ AIDS patients in South Africa: A 6-month follow-up study. J Psychosom Res 2006; 60 : 479-484

5. Nath A, Schiess N, Venkatesan A, Rumbaugh J, Sacktor N, McArthur J. Evolution of HIV dementia with HIV infection. International Review of Psychiatry 2008; 20(1): 2531

6. Sacktor N, Lyles R, Skolasky R, et al., and the Multicenter AIDS Cohort Study. HIVassociated neurologic disease incidence changes: Multicenter AIDS Cohort Study, 1990 - 1998. Neurology 200 1; 56: 257-260.

7. Freeman M, Patel V, Collins PY, Bertolote J. Integrating mental health in global initiatives for HIV/AIDS. Br J Psychiatry 2005; 187: 1-3.

8. Meade CS, Sikkema KJ. Psychiatric and psychosocial correlates of sexual risk behavio among adults with severe mental illness. Community Ment Health J 2007; 43(2): 153 169. Epub 2006 Dec 2

9. Treismana G, Kaplina A. Neurologic and psychiatric complications of antiretroviral agents. AIDS 2002: 16:1201-1215.

10. Joint United Nations Programme on HIV/AIDS (UNAIDS) and World Health Organization (WHO). AlDS epidemic update. 2007. http://data. unaids.org/pub/ EPISlides/2007/2007_epiupdate_en.pdf (accessed 10 September 2008).

11. Provincial Antiretroviral Monitoring and Evaluation Team. The Western Cape Antiretroviral Programme, Internal Monitoring Report 2006/07. Cape Town: Provincial Government of the Western Cape, 2007

12. HIV/AIDS Treatment Adherence, Health Outcomes and Cost Study Group. The HIV/ AIDS Treatment Adherence, Health Outcomes and Cost Study: conceptual foundations and overview. AIDS Care 2004; 16: Suppl 1, S6-S2 1

13. Patel V, Araya R, Chowdhary N, et al. Detecting common mental disorders in primary care in India: a comparison of five screening questionnaires. Psychological Medicine 2008, 38: 221-228

14. Goldberg D, Williams P. A User's Guide to the General Health Questionnaire. Windsor: nferNelson, 1988

15. Kessler RC, Andrews G, Colpe L, et al. Short screening scales to monitor population prevalences and trends in non-specific psychological distress. Psychol Med 2002; 32 : 959-976

16. Pence B, Gaynes B, Whetten K, Eron J, Ryder R, Miller W. Validation of a brief screening instrument for substance abuse and mental illness in HIV-positive patients. J Acquir Immune Defic Syndr 2005; 40: 434-444.

17. Myer L, Smit J, le Roux L, Parker S, Stein DJ, Seedat S. Common mental disorders among HIV-infected individuals in South Africa: prevalence, predictors and validation of brief psychiatric rating scales. AIDS Patient Care and STDs 2008; 22(2): 147-158.

18. Sacktor N, Wong M, Nakasuijac N, et al. The International HIV Dementia Scale: a new rapid screening test for HIV dementia. AIDS 2005; 19: 1367-1374. 